\title{
=ALERIDAD Del conocimiento previo a la elaboración conceptual: Un caso en educación primaria
}

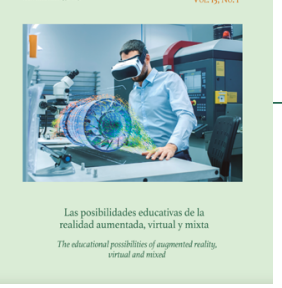

\author{
From prior knowledge to conceptual elaboration: A case in \\ primary education
}

(iD)

Yesney Bethencourt es docente contratada de la Universidad Pedagógica Experimental Libertador (Venezuela) (yesve75@hotmail.com) (https://orcid.org/0000-0002-5137-477X)

iD Aracelis Arana es docente asociado de la Universidad Pedagógica Experimental Libertador (Venezuela) (aracelisarana3@gmail.com) (https://orcid.org/0000-0003-3430-2640)

Recibido: 2019-01-30 / Revisado: 2019-11-19 / Aceptado: 2019-12-09 / Publicado: 2020-01-01

\section{Resumen}

En la Educación Primaria Venezolana (Ministerio del Poder Popular para la Educación -MPPE- 2007) se insertó el ambiente como un eje que integra las disciplinas del área de matemática y ciencias naturales, desde esta perspectiva el aprendizaje de este concepto mes fundamental para la comprensión de los conceptos científicos asociados. Con el objetivo de describir la dinámica cognitiva implicada en su aprendizaje se consideró necesario investigar el desarrollo del mismo en estudiantes de tercer grado, desde sus nociones previas hasta la conceptualización del ambiente como sistema complejo. El estudio se enmarcó en una Investigación de Campo, bajo un diseño de investigación-acción. A los fines de recabar la información necesaria de 20 informantes seleccionados, se les aplicó un cuestionario de respuesta abierta al inicio y al final del lapso. Para el análisis de la información se utilizó la triangulación temporal individual y de grupo, identificando así los operadores conceptuales utilizados y los aspectos convergentes entre ellos. Del análisis realizado emergieron cinco (5) categorías que reúnen todas las formas de conceptualizar el ambiente para el momento I y Cinco (5) categorías para el momento 2, de éstas, la última hace referencia a la visión sistémica-compleja en dónde se ubicaron II de los 20 informantes. Se concluye que la metódica empleada permitió contrastar los avances de los estudiantes en el desarrollo de sus conceptualizaciones y la dinámica cognitiva implicada.

Descriptores: Conocimiento, aprendizaje, conceptos, enseñanza, didáctica, educación.

\begin{abstract}
In the Venezuelan Primary Education (MPPE, 2007) the environment was inserted as an integrating axis that connects with the disciplines of the area of mathematics and natural sciences, from this perspective the learning of this concept is fundamental for the understanding of the associated scientific concepts. In order to describe the cognitive dynamics involved in their learning, it was considered necessary to investigate the development of the same in third grade students, from their previous notions to the conceptualization of the environment as a complex system. The study was framed in a Field Investigation, under an action research design. In order to gather the necessary information from 20 selected informants, an open response questionnaire was applied at the beginning and end of the period. For the analysis of the information, the individual and group temporal triangulation was used, identifying the conceptual operators used and the convergent aspects among them. From the analysis made five (5) categories emerged that bring together all the ways of conceptualizing the environment for the moment I and Five (5) categories for the
\end{abstract}

Forma sugerida de citar: Bethencourt, Y., \& Arana, A. (2020). Del conocimiento previo a la elaboración conceptual. Un caso en educación primaria. Alteridad, 15(1), 88-101. https://doi.org/10.17163/alt.v15n1.2020.07 
moment 2, of these, the latter makes reference to the systemic-complex vision where they were located II of the 20 informants. It is concluded that the method used allowed to contrast the advances of the students in the development of their conceptualizations and the cognitive dynamics involved.

Keywords: Knowledge, learning, concepts, teaching, didactics, education.

\section{Introducción}

El tema sobre el ambiente y su conservación es tan importante que la Organización de las Naciones Unidas (ONU, 2002, 2005) a través de la (UNESCO, 2012, 2015) insisten permanentemente en la inclusión obligatoria de los programas de Educación Ambiental para el desarrollo sostenible en todos los niveles del sistema educativo, a nivel mundial; con la finalidad de formar a los padres, maestros, obreros, patrones, empresarios y políticos hacia la consideración del ambiente como un sistema desde la perspectiva del enfoque complejo, que conlleve a una visión integradora de sus componentes sociales y naturales, a partir de la relación e interconexión de los procesos que en él se ejecutan. En tal sentido, es fundamental orientar la formación de los estudiantes hacia una visión del ambiente más cercana a su realidad, considerándose como un componente más de sus interacciones, que interviene con él, en su comunidad, su salud, su alimentación, sus enfermedades, su empleo, entre otros.

En atención a estas disposiciones, en el Currículo Nacional Bolivariano del Subsistema de Educación Primaria (MPPE, 2007), se incluyó como un eje integrador: "Ambiente y Salud Integral" para organizar e integrar los saberes (p. 20), que se conecta fundamentalmente con el Área de Aprendizaje "Matemática, Ciencias Naturales y Sociedad" (p. 57). Entre sus contenidos para el tercer grado, se plantea: "Noción de ambiente: observación e interpretación del ambiente en mi comunidad, región y país. Investigación sobre la Biosfera. Establecimiento de inferencias para determinar que la biosfera está conformada por seres vivos: humanos, animales, plantas y organismos microscópicos” (p. 59).

Desde estos requerimientos del currículo actual venezolano, cabría preguntarse: ¿Cuál es la noción de ambiente que tienen los estudiantes cuando inician el tercer grado de Educación Primaria? ¿Cómo lograr que esta noción de ambiente se desarrolle en función de los requerimientos institucionales y curriculares? ¿Será posible que los niños de tercer grado puedan desarrollar una conceptualización de ambiente como sistema complejo? Para darle respuesta a estas interrogantes las autoras consideraron necesario llevar a cabo un estudio de campo en la Unidad Educativa Nacional "Meregoto", ubicada en el Municipio Sucre del estado Aragua, a los fines de profundizar en cómo se desarrolla la noción de ambiente en estudiantes de tercer grado, a partir de la aplicación de un Proyecto de Aprendizaje diseñado con este fin.

\section{Objetivos de la investigación}

- Precisar la noción de ambiente que traen los estudiantes del tercer grado al inicio del curso, mediante la exteriorización de sus elaboraciones proposicionales.

- Diseñar un Proyecto de Aprendizaje a partir de las ideas e inquietudes aportadas por los estudiantes, en relación con su noción previa acerca del ambiente.

- Examinar el avance logrado por los estudiantes en el desarrollo de la noción de ambiente después de la aplicación del Proyecto de Aprendizaje.

- Develar la dinámica cognitiva implicada en el desarrollo del concepto de ambiente alcanzado por los estudiantes a partir de sus elaboraciones proposicionales.

\section{Bosquejo referencial}

Tal como se señala en párrafos anteriores, el concepto de ambiente conforma en el Currículo Nacional Bolivariano del Subsistema de Educación 
Primaria (MPPE, 2007) uno de los ejes integradores del Área de Aprendizaje "Matemática, Ciencias Naturales y Sociedad", y en el mismo aparecen los contenidos concernientes a la necesidad de desarrollar una noción de ambiente que vaya evolucionando desde primer grado como: "Noción de ambiente, construcción del concepto a partir de la identificación de los componentes del ambiente" (p. 34); en el segundo grado: "Noción de ambiente, concepto, identificación y descripción de los componentes del ambiente, el ambiente en mi comunidad" (p. 46); en el tercer grado: "Noción de ambiente: observación e interpretación del ambiente en mi comunidad, región y país, investigación sobre la Biósfera” (p. 59).

En la descripción anterior de los contenidos se aprecia cómo en torno a la noción de ambiente se van articulando diversos términos propios de las Ciencias Naturales: Aire, Agua, Suelo, Cuerpo Humano, Animales, Vegetales; también se incluye el estudio del ciclo del agua, el clima, la contaminación, entre otros, acrecentando su rango de acción y complejidad en la misma medida en que aumenta el grado; de manera que, la construcción y aprehensión de este concepto es esencial para la comprensión de la complejidad del ambiente y sus diferentes componentes. Por otra parte, es importante destacar mencionar que la nueva Ley Orgánica de Educación (2009) en su artículo 14 afirma que "la educación ambiental... son de obligatorio cumplimiento, en las instituciones y centros educativos oficiales y privados" (p. 10) y el artículo 15 numeral 5, reafirma como fines de la Educación: "Impulsar la formación de una conciencia ecológica para preservar la biodiversidad y la socio-diversidad, las condiciones ambientales y el aprovechamiento racional de los recursos naturales" (p. 12), con lo que el Estado legitima la importancia de la educación ambiental en Venezuela.

En este orden de ideas, distintos autores (Meira, 2006; Ochoa García, 2015; Alfonso Martínez, 2016), ante la globalización del desarrollo y su impacto sobre el ambiente han propuesto la necesidad de dotar a la población de una serie de nociones sobre cómo es y cómo funciona el ambiente para que las personas puedan en consecuencia actuar responsablemente en él. En este sentido, la autora ha realizado estudios de cómo se desarrollan y se procesan cognitivamente los conceptos científicos (Arana \& González, 2006; Arana, 2007a y 2012), en relación con el desarrollo del concepto de ambiente en contextos y situaciones escolares, se han monitoreado los conocimientos previos de los estudiantes, el procesamiento y evolución de los mismos a partir de las representaciones mentales que construyen, y que ellos exteriorizan mediante proposiciones escritas, dibujos, mapas conceptuales y entramados, estimándose la importante función mediadora del docente en la construcción del conocimiento.

En tal sentido, se considera que una de las condiciones fundamentales para el logro del éxito de cualquier reforma educativa radica en que los docentes generen un cambio en las estrategias educativas que planifican, por tal motivo esta investigación pretende contribuir a la transformación de la práctica educativa que se efectúa en las aulas de clase, desarrollando iniciativas didácticas para el Área de Ciencias Naturales y más específicamente a la pretensión de modelar las concepciones previas con la que los estudiantes llegan a la escuela, que según Meira (2006), normalmente son de carácter coloquial y superfluo, al consistir en acciones, percepciones y concepciones fragmentarias o aisladas.

Dentro de esta perspectiva, y tomando en cuenta los requerimientos que plantea durante los primeros tres años el Currículo Nacional Bolivariano (MPPE, 2007), y también las previstas en todos los convenios firmados en torno a la Educación Ambiental, con respecto a una noción del ambiente como sistema complejo, es fundamental orientar la formación de los estudiantes hacia una noción de ambiente más en su visión de complejidad (Arana, 2012), más cercana a la realidad, analizando sus componentes y cómo se relacionan con él, con su comunidad, con su salud, con su alimentación, con las enfermedades, el empleo y la pobreza (Unesco, 2015). 


\section{Metodología}

El estudio se enmarcó en una Investigación de Campo de carácter descriptivo, bajo el enfoque cualitativo de un diseño de Investigación Acción-Participante que según diversos autores (Barcedas, 2005; Becerra \& Moya, 2010; Colmenares, 2012) es una metodología apropiada cuando la opinión de los participantes de un programa es fundamental, o cuando es necesario considerar como viven, sienten y se expresan los protagonistas, en consecuencia se busca profundizar en el procesos de aprendizaje del concepto de ambiente, en estudiantes del tercer grado de Educación Primaria de la UEN “Meregoto" desde sus conocimientos previos hasta una concepción del ambiente como sistema complejo, analizando sus componentes, cómo se relacionan entre sí, consigo mismo y con su contexto. Para tal fin, se diseñó y ejecutó un proyecto de aprendizaje denominado: "Desarrollando mi noción de ambiente", con el propósito de implicar a los participantes en actividades dentro y fuera del aula a fin de ponerlos en contacto con el ambiente al cual están integrados. Al respecto, Colmenares (2012) establece que la participación de los involucrados son también investigadores y beneficiarios de los hallazgos y soluciones o propuestas, de manera que las investigadoras participaron como asesoras en todas las actividades que se ejecutaron, a fin de facilitar y orientar el aprendizaje de los estudiantes como protagonistas.

Asimismo, Bausela (2004) afirma que la investigación-acción se presenta como una metodología de investigación orientada hacia el cambio educativo que se configura como una espiral de ciclos de planificación, acción, observación y reflexión, es decir que el procedimiento de acción según Corral, Corral y Corral (2016) se desarrolla siguiendo un modelo en espiral, en ciclos sucesivos que incluyen diagnóstico, planificación, acción, observación y reflexiónevaluación. Este proceso de investigación es descrito con matizaciones diferentes según autores, variando en cuanto a su complejidad. La figura 1 muestra las principales fases:

Gráfico 1. Fases y secuencias de la metodología que se aplica desde la investigación-acción.

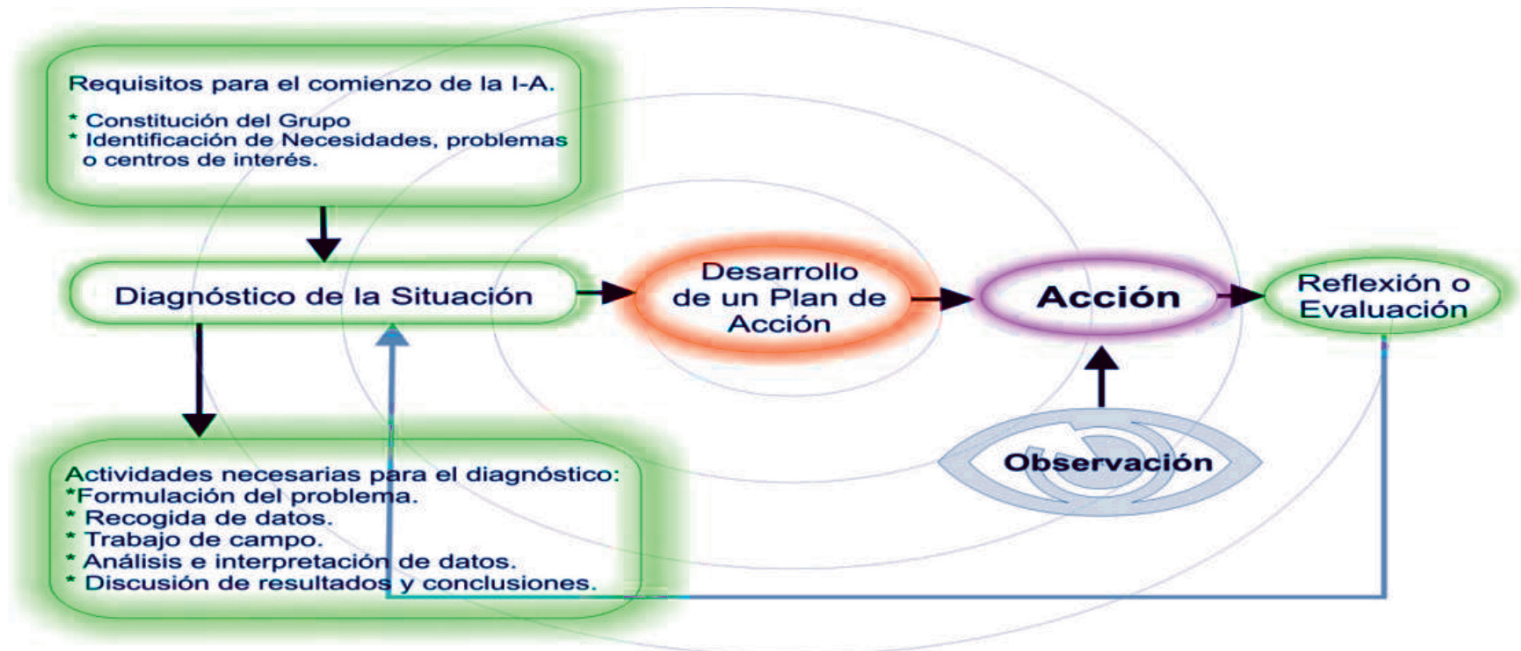

Fuente: Buendía y otros (2000, p. 297), adaptado por las autoras. 


\section{Informantes claves}

El estudio se llevó a cabo en dos secciones (C y D) de tercer grado en la UEN "Meregoto", donde se desempeña una de las autoras como maestra de tercer grado, debido a que en este nivel de la Educación Primaria los niños presentan competencias de lecto-escritura más desarrolladas, las cuales permiten aplicar las técnicas e instrumentos propios de este tipo de investigación. Los informantes claves se seleccionaron al finalizar el curso, una vez revisada toda la información recabada, bajo el criterio de los estudiantes que ejecutaron todas las actividades de evaluación, ya que esta condición era necesaria para obtener una información consistente sobre el tema en estudio, el grupo de informantes quedó conformado por 20 informantes (diez en cada sección), de un total de 50 estudiantes.

\section{Técnicas e Instrumentos de acopio de información}

Por las características del fenómeno que se estudió y de acuerdo con Martínez (1996 y 1999) en términos de los intereses del proceso investigativo se utilizó como técnica de recogida de información la observación no participante, mediante la aplicación de un cuestionario de preguntas abiertas sometido a evaluación de expertos (Hernández, Fernández \& Baptista, 2003), con el propósito de indagar sobre las ideas previas que tienen acerca del ambiente al inicio y al final del curso para comparar el avance logrado por los estudiantes durante la implementación del proyecto de aprendizaje. Dicho instrumento fue estructurado en base a cuatro ejercicios dirigidos a obtener información sobre: a) la definición del concepto de ambiente (representación proposicional), b) Qué acciones realizan para conservar el ambiente, c) representación pictórica del concepto y d) representación gráfica del mismo.

\section{Diseño del Plan de Acción y Ejecución del proyecto}

La concepción de ambiente a desarrollar en este proyecto, es una visión sistémica compleja del mismo, donde el conocimiento y la acción aplicados a los procesos biológicos estén indisolublemente ligados a los procesos sociales e históricos, es decir incluir toda la complejidad de las relaciones que se establecen entre los seres humanos y los elementos socio-naturales. Adoptando este punto de vista, según Pacheco (2005), se puede desarrollar una redefinición de este concepto conducente a un cambio de su significado que permitiría el surgimiento de nuevas herramientas conceptuales y metodológicas en la adaptación e intervención del ambiente por parte de la especie humana. Una de las claves con las que se trabajó este proyecto fue partir de las preguntas que los estudiantes se hacen a sí mismos acerca del tema en estudio. Para la elaboración y diseño de las estrategias de enseñanza-aprendizaje del proyecto educativo se tomaron en consideración los planteamientos de Arana (2007b y 2010) sobre la planificación de estrategias integradoras. La ejecución del proyecto se llevó a cabo en el último trimestre del período escolar 2012/2013, específicamente desde el veinte (20) de mayo al veintiséis (26) de junio del 2013, con un alcance de cuatro semanas.

\section{Organización y metódica de análisis de la información recabada}

Una vez cumplidas todas las fases del proyecto de aprendizaje y finalizado el curso, se procedió a organizar la información recabada mediante la aplicación del cuestionario:

- Al inicio del curso, para indagar sobre que noción tienen los informantes acerca del concepto de ambiente. En función de estos resultados y considerando sus inquietudes, se diseñó el Proyecto de Aprendizaje para 
promover un avance de la noción de ambiente como sistema complejo, que permitiera el desarrollo de una visión integral de sus componentes y la inclusión del ser humano, como un elemento más de ese sistema.

- Después de ejecutado el Proyecto de Aprendizaje se les aplicó el mismo cuestionario, a fin de contrastar el avance logrado por los informantes en relación con el concepto en estudio y poder analizar su progreso con respecto al concepto institucional de referencia (Arana, 2008).

Seguidamente se ordenaron las definiciones dadas por cada informante, para el concepto en estos dos momentos y se procedió a realizar un análisis de comparación continua (Glaser \& Strauss, 1967; Osses, Sánchez, \& Ibañes, 2006), para detectar todas las formas de expresar el concepto de ambiente por los informantes (triangulación temporal de nivel individual) y se extrajeron los operadores conceptuales (conjunto de atributos de criterio que permiten reconocer y expresar lo que es o su significado) utilizados en la expresión de sus proposiciones para cada sección (Ver cuadro 1). Posteriormente, en función de estos operadores extraídos, se comparan entre sí las definiciones dadas por ambos grupos (triangulación interactiva de nivel colectivo), con la finalidad de encontrar patrones o regularidades entre ellos. De acuerdo con Pérez (1998), la triangulación es un procedimiento de análisis que implica la reunión de datos y métodos sobre un mismo tema o problema, que se recogen desde puntos de vista distintos, a fin de realizar comparaciones múltiples de un fenómeno único, de un grupo, en diferentes momentos, utilizando diversas perspectivas. Al respecto, Barcedas (2005) coincide en afirmar que triangular es: "utilizar el control cruzado de informaciones, buscando compensar la debilidad de la metodología con la complementariedad y convergencia de distintos procedimientos, sometiendo al control cruzado los puntos de vista de diferentes participantes frente a un mismo tema" (p. 34).

\section{Análisis integrado de los resultados por sección.}

\section{Sección C}

Seguidamente se ofrecen los resultados obtenidos de la aplicación del instrumento, a la interrogante $\mathrm{N}^{\circ} 1$, en los dos momentos previstos $y$ el análisis integrado a las elaboraciones proposicionales exteriorizadas por los informantes de la sección C, en función de los operadores conceptuales utilizados (Ver Cuadro 1).

A primera vista, se aprecia que todos los informantes enriquecieron y mejoraron sus definiciones acerca del ambiente en la segunda aplicación del cuestionario, lo cual se esperaba después de las vivencias y estrategias de aprendizaje realizadas durante la ejecución del proyecto.

Examinando con más detalles las respuestas dadas en la primera evaluación (M1), se observa que cuatro de los informantes $(\mathrm{C} 2, \mathrm{C} 8, \mathrm{C} 9$ y C10) expresan una visión antropocéntrica del ambiente "todo lo que nos rodea", otros cinco informantes (C1, C3, C4, C6 y C7) se orientan hacia una visión ligada a la vida, lugar o espacio para vivir, alguno de los cuales (C1 y C6) resaltan que debe estar limpio. Y una informante (C5) manifiesta una visión estética "El ambiente es hermoso" que la coloca en la categoría de preconcepto.

En términos generales, ninguna de estas definiciones se aproxima al concepto de ambiente asociado con el enfoque sistémico (Concepto Institucional de Referencia), lo cual es natural porque son nociones que han estructurado a partir de sus vivencias, tal como lo señala Vigotsky (citado por Benbenaste, Luzzi \& Costa, 2007) el empleo de estas definiciones dan cuenta de un conocimiento cotidiano, estas nociones se apoyan en la manera básica, familiar, conque el sujeto efectiviza su entorno, es como él le da significado al mundo. 
Cuadro 1. Elaboraciones proposicionales exteriorizadas por los informantes (Sección C) a la interrogante $N^{\circ} 1$

\section{Define con tus propias palabras ¿Qué es para ti el ambiente?}

\begin{tabular}{|c|c|c|c|c|}
\hline $\begin{array}{l}\text { Informan- } \\
\text { tes Claves }\end{array}$ & $\begin{array}{c}\text { Evaluación } 1 \text { (M1) } \\
\text { Conocimientos Previos }\end{array}$ & $\begin{array}{l}\text { Operador } \\
\text { conceptual }\end{array}$ & $\begin{array}{l}\text { Evaluación } 2 \text { (M2) } \\
\text { Evaluación Final }\end{array}$ & $\begin{array}{l}\text { Operador } \\
\text { Conceptual }\end{array}$ \\
\hline C1 & $\begin{array}{l}\text { Amor, paz, porque es impecable } \\
\text { y sin basura, y porque hay ani- } \\
\text { males que necesitan el ambiente } \\
\text { limpio para vivir. }\end{array}$ & $\begin{array}{l}\text { Amor, paz am- } \\
\text { biente limpio } \\
\text { para vivir }\end{array}$ & $\begin{array}{l}\text { Es la interacción entre las cosas de la } \\
\text { naturaleza, el hombre y la sociedad, } \\
\text { ejemplo: el niño que agarra el autobús } \\
\text { para ir a la escuela acompañado de } \\
\text { su mamá y de la mascota. }\end{array}$ & $\begin{array}{l}\text { Interacción, } \\
\text { Naturaleza, } \\
\text { hombre y } \\
\text { sociedad. }\end{array}$ \\
\hline $\mathrm{C} 2$ & $\begin{array}{l}\text { Es lo que nos da la vida, lo que } \\
\text { nos rodea, y todos los elementos } \\
\text { de este planeta, como el agua, el } \\
\text { aíre, el fuego y la tierra. }\end{array}$ & $\begin{array}{l}\text { Lo que nos da } \\
\text { la vida, lo que } \\
\text { nos rodea, } \\
\text { todos los } \\
\text { elementos de } \\
\text { este planeta }\end{array}$ & $\begin{array}{l}\text { Es la interacción de elementos tales } \\
\text { como la hidrósfera, litósfera y atmós- } \\
\text { fera para que haya condiciones para } \\
\text { la vida de animales, plantas y seres } \\
\text { humanos. }\end{array}$ & $\begin{array}{l}\text { Interacción de } \\
\text { elementos, } \\
\text { hidrósfera, } \\
\text { litósfera, con- } \\
\text { diciones para } \\
\text { la vida }\end{array}$ \\
\hline C3 & $\begin{array}{l}\text { Vida, aire, sin él no podemos vivir, } \\
\text { respirar, para mi parece la selva. }\end{array}$ & $\begin{array}{l}\text { Vida, aire, no } \\
\text { podemos vivir } \\
\text { sin él }\end{array}$ & $\begin{array}{l}\text { Es un conjunto de elementos que se } \\
\text { relacionan entre sí (animales, plantas, } \\
\text { hombre y cosas elaboradas por los } \\
\text { humanos). }\end{array}$ & $\begin{array}{l}\text { Conjunto de } \\
\text { elementos que } \\
\text { se relacionan. }\end{array}$ \\
\hline $\mathrm{C} 4$ & $\begin{array}{l}\text { Son los animales, el bosque, el } \\
\text { aíre, puro y limpio, los árboles, el } \\
\text { ambiente es una fuente de vida } \\
\text { para los animales y las personas. }\end{array}$ & $\begin{array}{l}\text { Es una fuente } \\
\text { de vida. }\end{array}$ & $\begin{array}{l}\text { Son los animales (mi mascota, el ele- } \\
\text { fante Lucky), las personas (mi mamá, } \\
\text { papá, mis compañeritos), la tecnología } \\
\text { (casas, carros, la escuela, mi T.V.) } \\
\text { que actúan entre sí para mantenernos } \\
\text { vivos. }\end{array}$ & $\begin{array}{l}\text { Actúan entre sí } \\
\text { para mante- } \\
\text { nernos vivos. }\end{array}$ \\
\hline C5 & $\begin{array}{l}\text { Es para mí lo mejor que me ha } \\
\text { dado la vida y el ambiente para } \\
\text { mi es hermoso. }\end{array}$ & Hermoso & $\begin{array}{l}\text { Son las personas, los edificios, los ani- } \\
\text { males, las plantas, la mesa, las sillas, } \\
\text { las puertas, el ventilador, la escuela, la } \\
\text { iglesia, es todo lo que nos rodea. }\end{array}$ & $\begin{array}{l}\text { Personas, } \\
\text { edificios, } \\
\text { animales, es } \\
\text { todo lo que } \\
\text { nos rodea. }\end{array}$ \\
\hline C6 & $\begin{array}{l}\text { El ambiente es el lugar donde } \\
\text { podemos vivir, el ambiente es } \\
\text { grande, tan grande que se puede } \\
\text { ver, si el ambiente no estuviera } \\
\text { limpio no podríamos vivir. }\end{array}$ & $\begin{array}{l}\text { Lugar para } \\
\text { vivir, si no } \\
\text { está limpio } \\
\text { no podríamos } \\
\text { vivir. }\end{array}$ & $\begin{array}{l}\text { Son los elementos que interactúan } \\
\text { para mantener la vida, pero el hombre } \\
\text { daña y pone en peligro al planeta. }\end{array}$ & $\begin{array}{l}\text { Elementos que } \\
\text { interactúan } \\
\text { entre sí para } \\
\text { mantener la } \\
\text { vida. }\end{array}$ \\
\hline C7 & $\begin{array}{l}\text { Es un fenómeno ambiental. Un } \\
\text { lugar natural en que todos los } \\
\text { humanos son felices y creen que } \\
\text { es genial para la tierra, un lugar } \\
\text { para vivir. }\end{array}$ & $\begin{array}{l}\text { Fenómeno } \\
\text { ambiental, } \\
\text { un lugar para } \\
\text { vivir. }\end{array}$ & $\begin{array}{l}\text { Es un sistema que incluye a todos } \\
\text { los seres vivos que se desarrollan en } \\
\text { la Biosfera, que hay que cuidar para } \\
\text { mantener la vida. }\end{array}$ & $\begin{array}{l}\text { Sistema, Biós- } \\
\text { fera, cuidar } \\
\text { para mantener } \\
\text { la vida. }\end{array}$ \\
\hline C8 & $\begin{array}{l}\text { El ambiente es todo lo que nos } \\
\text { rodea. }\end{array}$ & $\begin{array}{l}\text { Todo lo que } \\
\text { nos rodea. }\end{array}$ & $\begin{array}{l}\text { Es la interacción que sucede entre los } \\
\text { seres humanos, los seres vivos y las } \\
\text { comunidades. }\end{array}$ & $\begin{array}{l}\text { Interacción } \\
\text { entre seres } \\
\text { humanos, } \\
\text { seres vivos y } \\
\text { comunidades }\end{array}$ \\
\hline C9 & Es todo lo que nos rodea. & $\begin{array}{l}\text { Todo lo que } \\
\text { nos rodea }\end{array}$ & $\begin{array}{l}\text { Son las personas, las plantas, los } \\
\text { animales, las cosas creadas por el } \\
\text { hombre y Dios. }\end{array}$ & $\begin{array}{l}\text { Las cosas } \\
\text { creadas por } \\
\text { el hombre y } \\
\text { Dios. }\end{array}$ \\
\hline C10 & $\begin{array}{l}\text { Es todo lo que nos rodea: las plan- } \\
\text { tas, los animales, el aíre, el río. }\end{array}$ & $\begin{array}{l}\text { Todo lo que } \\
\text { nos rodea }\end{array}$ & $\begin{array}{l}\text { El ambiente es un hogar para los ani- } \\
\text { males, las plantas, y los seres vivos, } \\
\text { es muy importante para mi mantenerlo } \\
\text { limpio para después no enfermarnos. }\end{array}$ & $\begin{array}{l}\text { Hogar, impor- } \\
\text { tante mante- } \\
\text { nerlo limpio. }\end{array}$ \\
\hline
\end{tabular}


Ya para la segunda evaluación (M2) se puede apreciar un avance de los informantes, hacia niveles de mayor elaboración, utilizando operadores conceptuales propios del enfoque sistémico, tales como: sistema, interacciones, conjunto de elementos que se relaciones entre sí, elementos que interactúan entre sí, que denotan una aproximación más cercana al concepto institucional que se desea desarrollar en ellos. Es el caso de los informantes (C1, C2, C3, C6, C7 y C8). A partir de este análisis emergieron seis categorías que reúnen todas las formas y significados empleados por estos informantes en su conceptualización sobre el ambiente (Ver Cuadro 2), para ambos momentos.

Cuadro 2. Comparación de las categorías emergentes en el diagnóstico (M1) y en la evaluación final (M2) en la sección C

\begin{tabular}{|l|c|c|c|c|}
\hline \multirow{2}{*}{\multicolumn{1}{|c|}{ Categorías }} & \multicolumn{2}{c|}{ Diagnóstico } & \multicolumn{2}{c|}{ Evaluación Final } \\
\cline { 2 - 5 } & Informante & Frecuencia & Informante & 0 \\
\hline Preconcepto & C5 & 1 & --- & 1 \\
\hline Visión Mágico Religiosa & ---- & & C9 & 1 \\
\hline Visión Antropocéntrica & C2, C8, C9, C10 & 4 & C5 & 0 \\
\hline Visión fuente de Vida & C3, C4 & 2 & ---- & 0 \\
\hline Visión espacio de Vida & C7 & 1 & ---- & 1 \\
\hline Visión conservacionista & C1, C6 & 2 & C10 & 7 \\
\hline Visión Sistémica - Compleja & ------ & 0 & C1, C2, C3, C4, C6, C7, C8 \\
\hline
\end{tabular}

Ya obtenidas las categorías fueron ordenadas cronológicamente en función de su aproximación al concepto institucional de referencia más actualizado (Arana, 2008), se puede apreciar en el cuadro 2, que seis de los informantes (C1, C2 C3, C6, C7 y C8) avanzaron hacia niveles de mayor complejidad en sus definiciones, expresando una terminología más asociada al concepto de ambiente como sistema desarrollado en el proyecto de aprendizaje, lo que pone en evidencia el progreso conceptual experimentado por los informantes.

Es interesante el caso del informante C4, tiene una visión inicial del ambiente como fuente de vida, luego introduce elementos socio-tecnológicos como parte del ambiente y acota: "que actúan entre sí para mantenernos vivos”, es decir se orienta hacia la visión sistémica- compleja, pero todavía incompleta.

Asimismo, tenemos el caso del informante C9, que se moviliza de una visión antropocéntrica a una mágico-religiosa, lo cual no deja de ser contradictorio, considerando que la temática religiosa no fue abordada durante la realización del proyecto. Finalmente, se puede concluir que nueve de los diez informantes avanzaron hacia niveles de mayor complejidad en la elaboración de sus definiciones.

\section{Sección D}

A continuación se presentan en el cuadro 3, los resultados y análisis de las respuestas dadas por los informantes de la sección D. 
Cuadro 3. Elaboraciones proposicionales exteriorizadas por los informantes (Sección D) a la interrogante $N^{\circ} 1$

\section{Define con tus propias palabras ¿Qué es para ti el ambiente?}

\begin{tabular}{|c|c|c|c|c|}
\hline $\begin{array}{l}\text { Informantes } \\
\text { Claves }\end{array}$ & $\begin{array}{c}\text { Evaluación } 1 \\
\text { Conocimientos Previos }\end{array}$ & $\begin{array}{l}\text { Operador- } \\
\text { conceptual }\end{array}$ & $\begin{array}{l}\text { Evaluación } 2 \\
\text { Evaluación Final }\end{array}$ & $\begin{array}{l}\text { Operador } \\
\text { Conceptual }\end{array}$ \\
\hline D1 & $\begin{array}{l}\text { Las flores, los animales, } \\
\text { el río, la laguna, la gra- } \\
\text { ma, los pájaros. }\end{array}$ & $\begin{array}{l}\text { Elementos } \\
\text { naturales }\end{array}$ & $\begin{array}{l}\text { Es donde se desenvuelven todos los se- } \\
\text { res vivos, dentro de la esfera de la vida, } \\
\text { pero hay que cuidarla o todos moriremos. }\end{array}$ & $\begin{array}{l}\text { Desenvuelven } \\
\text { los seres vivos, } \\
\text { esfera de la } \\
\text { vida. }\end{array}$ \\
\hline D2 & $\begin{array}{l}\text { El ambiente es todo lo } \\
\text { que nos rodea. }\end{array}$ & $\begin{array}{l}\text { Todo lo que } \\
\text { nos rodea }\end{array}$ & $\begin{array}{l}\text { Es muy importante, sin él no es posible } \\
\text { la vida, tenemos que cuidarlo para tener } \\
\text { un mejor ambiente. }\end{array}$ & $\begin{array}{l}\text { No es posible la } \\
\text { vida, cuidarlo. }\end{array}$ \\
\hline D3 & $\begin{array}{l}\text { El ambiente es cuidar los } \\
\text { árboles, los animales y } \\
\text { las flores. }\end{array}$ & Cuidar & $\begin{array}{l}\text { Es todo lo que nos rodea como los seres } \\
\text { humanos, los animales, los edificios, y lo } \\
\text { tenemos que cuidar. }\end{array}$ & $\begin{array}{l}\text { Todo lo que nos } \\
\text { rodea, cuidar. }\end{array}$ \\
\hline D4 & $\begin{array}{l}\text { Es todo lo que nos ro- } \\
\text { dea: plantas, animales, } \\
\text { pájaros, árboles, luces, } \\
\text { casas, gente. }\end{array}$ & $\begin{array}{l}\text { Todo lo que } \\
\text { nos rodea }\end{array}$ & $\begin{array}{l}\text { El ambiente para mí es un hogar para los } \\
\text { animales y los seres vivos, y es muy impor- } \\
\text { tante porque gracias a él estamos vivos. }\end{array}$ & $\begin{array}{l}\text { Hogar, estamos } \\
\text { vivos. }\end{array}$ \\
\hline D5 & Es todo lo que nos rodea. & $\begin{array}{l}\text { Todo lo que } \\
\text { nos rodea }\end{array}$ & $\begin{array}{l}\text { Es el medio donde me desenvuelvo, la } \\
\text { escuela, la casa, la playa, el zoológico, y } \\
\text { que debo mantener limpio. }\end{array}$ & $\begin{array}{l}\text { Medio donde } \\
\text { desenvuelvo, } \\
\text { mantener limpio. }\end{array}$ \\
\hline D6 & $\begin{array}{l}\text { Lo que nos rodea: plan- } \\
\text { tas, árboles y montañas. }\end{array}$ & $\begin{array}{l}\text { Lo que nos } \\
\text { rodea }\end{array}$ & $\begin{array}{l}\text { Son los elementos de la naturaleza (ani- } \\
\text { males, plantas) y sociales (personas) } \\
\text { que interaccionan entre sí, pero está en } \\
\text { peligro por el hombre. }\end{array}$ & $\begin{array}{l}\text { Elementos, in- } \\
\text { teracción entre } \\
\text { sí, peligro por el } \\
\text { hombre. }\end{array}$ \\
\hline D7 & Es todo lo que nos rodea. & $\begin{array}{l}\text { Todo lo que } \\
\text { nos rodea }\end{array}$ & $\begin{array}{l}\text { Es la interacción de la naturaleza, los } \\
\text { seres humanos y la sociedad, pero cual- } \\
\text { quier desequilibrio creado por los seres } \\
\text { humanos puede amenazar toda la vida. }\end{array}$ & $\begin{array}{l}\text { Interacción de } \\
\text { la naturaleza, } \\
\text { seres humanos } \\
\text { y la sociedad, } \\
\text { desequilibrio }\end{array}$ \\
\hline D8 & Es todo lo que nos rodea. & $\begin{array}{l}\text { Todo lo que } \\
\text { nos rodea }\end{array}$ & $\begin{array}{l}\text { Es la interacción de los elementos pre- } \\
\text { sentes en la naturaleza, y los creados } \\
\text { por el hombre, si uno desaparece pone } \\
\text { en peligro a los otros, ejemplo: los osos } \\
\text { necesitan el hielo para vivir, nosotros de- } \\
\text { pendemos de las plantas, de los anima- } \\
\text { les y del agua para vivir. }\end{array}$ & $\begin{array}{l}\text { Interacción de } \\
\text { los elementos, } \\
\text { desaparece, de- } \\
\text { pendemos para } \\
\text { vivir. }\end{array}$ \\
\hline D9 & $\begin{array}{l}\text { El ambiente natural es } \\
\text { todo lo que nos rodea. }\end{array}$ & $\begin{array}{l}\text { Todo lo que } \\
\text { nos rodea. }\end{array}$ & $\begin{array}{l}\text { Es todo con lo que interactuamos los hu- } \\
\text { manos y del cual dependemos para vivir. }\end{array}$ & $\begin{array}{l}\text { Todo con lo que } \\
\text { interactuamos, } \\
\text { dependemos } \\
\text { para vivir. }\end{array}$ \\
\hline D10 & Es todo lo que nos rodea. & $\begin{array}{l}\text { Todo lo que } \\
\text { nos rodea }\end{array}$ & $\begin{array}{l}\text { Los elementos del ambiente, como el } \\
\text { agua, el aire, el suelo, las plantas, las per- } \\
\text { sonas, la sociedad, son un conjunto, que } \\
\text { se relacionan entre sí, para vivir mejor. }\end{array}$ & $\begin{array}{l}\text { Conjunto que se } \\
\text { relacionan entre } \\
\text { sí, vivir mejor. }\end{array}$ \\
\hline
\end{tabular}


$\mathrm{Al}$ igual que en el grupo anterior todos los informantes enriquecieron sus definiciones acerca del ambiente en la segunda aplicación del cuestionario, y de hecho esto se evidencia fácilmente, ya que, en su mayoría (8) de estos informantes expresaron al inicio una noción del ambiente antropocéntrica "todo lo que nos rodea", ligada en algunos casos a la visión naturalista (D4, D6, D9). Por otro lado, el informante D1, sólo alude a elementos naturales y el D3 señala que el ambiente es cuidar los elementos naturales, en ambos casos no muestran una proposición conceptual, se pueden catalogar como preconceptos.

No obstante, en el segundo momento de evaluación se nota un enriquecimiento mayor en los operadores que utilizan para definir el concepto. Como se muestra en el cuadro 3, en los primeros cinco informantes las elaboraciones proposicionales se movilizan hacia la consideración del ambiente como espacio de vida (D1, D2, D4, D5) y que debemos cuidarlo (D2, D3, D5), dejando atrás, en su mayoría la visión antropocéntrica, salvo el caso D3, denotando la inclusión de elementos sociales y de sí mismo como partes del ambiente.

Seguidamente se aprecia que las conceptualizaciones de los últimos cinco informantes se aproximaron más a la concepción institucional de referencia, especialmente los informantes D6, D7, D8; que lo describen como la interacción entre elementos naturales, humanos y sociales o el D10 "conjunto de elementos naturales, las personas, la sociedad, que se relacionan entre sî" (parafraseado) obsérvese el avance con respecto a sus nociones previas. Destaca el informante D8, cuando agrega: “...si uno desaparece pone en peligro a los otros", también los informantes D6 y D7 argumentan la amenaza que representa el ser humano para el equilibrio de este sistema, es decir toman conciencia de su responsabilidad ante la problemática ambiental.

Ahora bien, el informante D9, aun cuando utiliza el operador "interacción", sigue manteniendo una visión antropocéntrica al expresar: "Es todo con lo que interactuamos los humanos". A continuación, en el cuadro 4 se muestran las categorías que emergieron del análisis anterior.

Obsérvese que las elaboraciones proposicionales de todos los informantes se movilizan desde nociones muy simples a proposiciones conceptuales más elaboradas, más ricas en términos de los operadores conceptuales que utilizan y también a un nivel de mayor complejidad. El hecho de que surgiera al final la categoría sistémica-compleja, evidencian un progreso por parte de alguno de los informantes, desde sus ideas previas hacia concepciones asociadas con los aportes científicos propuestos institucionalmente, de manera que se puede afirmar que las actividades pedagógicas realizadas a lo largo del proyecto de aprendizaje fueron significativas para el logro de los objetivos propuestos, sobre todo la inclusión del ser humano, como un elemento más de ese sistema, con lo cual se va propiciando la formación integral, la visión y el pensamiento holístico en el educando que les permita interpretar su realidad, desde una nueva perspectiva.

Cuadro 4. Comparación de las categorías obtenidas en el diagnóstico y en la evaluación final en la sección D

\begin{tabular}{|l|l|l|l|l|}
\hline \multirow{2}{*}{ Categorías } & \multicolumn{1}{c|}{ Informante } & Frecuencia & Informante & Frecuencia \\
\cline { 2 - 5 } & D1, D3 & 2 & ---- & $-\cdots$ \\
\hline Preconcepto & D2, D4, D5, D6, D7, D8, D9, D10 & 8 & D3, D9 & 2 \\
\hline Visión Antropocéntrica & ---- & --- & D1, D2, D4, D5 & 4 \\
\hline Visión espacio de Vida & ----- & --- & D6, D7, D8, D10 & 4 \\
\hline Visión Sistémica-compleja & & & \\
\hline
\end{tabular}




\section{Discusión y conclusiones}

En síntesis, del estudio realizado se desprenden diversas proposiciones que aportan interesantes conclusiones con relación al aprendizaje de los conceptos y que se enumeran a continuación:

1. La inclusión del ambiente como eje integrador en el Plan de Estudios de Educación Primaria promueve diversas oportunidades de aprendizaje basado en proyectos educativos, esta es una de las estrategias fundamentales para que los niños comprendan el funcionamiento de los sistemas socio-naturales que posibilitan una visión más amplia de su realidad, de sus problemas y mejores soluciones, desde un abordaje interdisciplinario y transdiciplinario, tal como lo exige el Currículo Nacional Bolivariano (MPPE, 2007).

2. La enseñanza-aprendizaje de los conceptos científico amerita de estrategias didácticas integradoras dirigidas a la activación, en los estudiantes, de procesos mentales de observación, asociación, comparación, elaboraciones conceptuales, entre otros; que posteriormente puedan ser exteriorizadas mediante definiciones, dibujos, gráficos, procesadores de información como mapas mentales, mapas conceptuales y otros, a fin de que el docente pueda cotejar el avance progresivo en el desarrollo de los mismos, desde el inicio del curso.

3. Se pudo corroborar que los conocimientos previos expresados por los informantes, en su mayoría (12 de 20) guardan relación con una noción ligada a la concepción antropocéntrica y otros (3 de 20) a una concepción ligada a la posibilidad de vida o espacio de vida. La actitud conservacionista sólo se apreció en dos (2 de 20) de los informantes, lo cual coincide con otras investigaciones realizadas (Arana \& González, 2006), en niveles superiores de la educación, y que evidencia la concepción de los seres humanos como eje central del ambiente.

4. Al examinar el avance logrado por los informantes, se observó que en ambos grupos se aprecia en todos los informantes un incremento en la cantidad de operadores conceptuales que utilizan para elaborar sus definiciones entre (M1 y M2), es decir, se produce un enriquecimiento conceptual progresivo (Arana, 2007a) que va configurando un mayor significado en la red cognitiva de cada uno de los informantes, lo cual se evidencia por la coherencia en sus expresiones escritas, y los ejemplos que refieren en algunos casos.

5. De lo anterior se desprende que los informantes movilizaron sus elaboraciones conceptuales hacia niveles de mayor complejidad, aproximándose progresivamente al concepto institucional de referencia. Tal como lo señalan numerosos autores (Villegas \& González, 2005; Moreno, 2012; Rey-Herrera \& Candela, 2013) en relación con la construcción de estructuras cognitivas que incrementan la capacidad interpretativa del entorno, así también se nota el desarrollo de una actitud conservacionista (10 de 20 informantes). Ver cuadro 5, sombreado y con $\left(^{*}\right)$.

6. En términos generales, la dinámica cognitiva implicada en el aprendizaje conceptual pareciera obedecer a un movimiento de avance cuya trayectoria es progresiva en términos de su complejidad (Ver cuadro 5). Esta dinámica se aprecia mejor cuando se realizan más de dos evaluaciones, tal como se observa en investigaciones realizadas por la autora con anterioridad. Seguidamente se presenta una interpretación esquemática de la trayectoria:

6.1. Obsérvese que los informantes ubicados en la categoría de preconceptos $(\leftrightarrow)$ ) avanzan a categorías interme- 
dias, visión antropocéntrica (C5 y D3) y espacio de vida (D1).

6.2. Los informantes ubicados en la categoría antropocéntrica $(\leftarrow \cdot \cdots \cdot \cdot \rightarrow)$ avanzan a la categoría espacio de vida $\left(\mathrm{C} 10^{*}\right.$ $\left.\mathrm{D} 2^{\star}, \mathrm{D} 4, \mathrm{D} 5^{\star}\right)$ y visión sistémica com- pleja $\left(\mathrm{C} 2, \mathrm{C} 8 \mathrm{C} 3, \mathrm{C} 4, \mathrm{C}^{\star}{ }^{\star} \mathrm{C} 1, \mathrm{C} 6 \mathrm{D} 6^{\star}\right.$, $\left.\mathrm{D}^{\star}, \mathrm{D} 8^{\star} \mathrm{D} 10\right)$.

6.3. Todos los informantes ubicados en las categorías visión fuente de vida y conservacionista $(\ll \cdots \cdots . . . \rightarrow$ ) avanzan a la categoría visión sistémica-compleja $\left(\mathrm{C} 3, \mathrm{C} 4, \mathrm{C} 7^{\star}\right.$ y C1, $\left.\mathrm{C} 6^{\star}\right)$.

\section{Cuadro 5. Dinámica cognitiva}

\begin{tabular}{|c|c|c|}
\hline Categorías iniciales & Movilidad cognitiva & Categorías finales \\
\hline $\begin{array}{l}\text { Preconceptos } \\
\text { C5, D3 }<1--1,-1 \\
\text { D1, <- }\end{array}$ & & $\begin{array}{l}\text { Visión Mágico religiosa } \\
\text { C9 }\end{array}$ \\
\hline 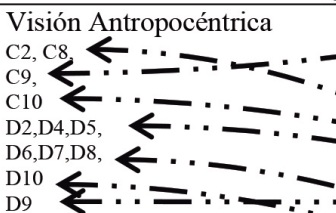 & & $\begin{array}{l}>_{\mathrm{C} 5, \mathrm{D} 3^{*}} \\
\text { Visión Antropocéntrica }\end{array}$ \\
\hline $\begin{array}{l}\text { Visión Fuente de vida o } \\
\text { espacio de vida } \\
\text { C3, C4, C7 }\end{array}$ & & $\begin{array}{l}\text { Visión Fuente de vida /espacio de vida } \\
\text { D1* } \\
\text { C10* } \\
\text { D2*, D4, D5* }\end{array}$ \\
\hline \multicolumn{3}{|l|}{ Visión Conservacionista } \\
\hline & & $\begin{array}{l}\text { Visión Sistémica compleja } \\
\text { C2, C8 } \\
\text { D6*, D7*,D8* } \\
\text { D10 } \\
\text { C3, C4, C7* } \\
\mathrm{C} 1, \mathrm{C}^{*}\end{array}$ \\
\hline
\end{tabular}

* Los informantes marcados con el asterisco denotan la actitud conservacionista.

7. La metódica de análisis aplicada resultó adecuada a los fines de develar la dinámica cognitiva implicada en el desarrollo de los conceptos, lo cual resulta importante para la investigación educativa y para los docentes, ya que en la enseñanza es fundamental saber cómo se desarrollan los conceptos en los aprendices, analizar cómo se reorganiza el conocimiento previo con la integración de nueva información y cómo se procesan esas nuevas aportaciones en ellos.

\section{Referencias bibliográficas}

Alfonso Martínez, O. (2016). Globalización y el impacto ambiental. (http://bit.ly/348976S).

Arana, A., \& González, F. (2006). Enriquecimiento conceptual progresivo. Una explicación teórica del proceso de desarrollo de los conceptos científicos. Revista de Pedagogía, UCV, XXVII(79), 193-230. (http://bit.ly/2S5qoep)

Arana, A. (2007a). Representando la complejidad ambiental: dos estudios de caso. Investigación $y$ postgrado, 22(1), 13-58. ISSN 1316-0087. (http://bit.ly/2M8ChNb) 
Arana, A. (2007b). Entramados Ambientales: Un modelo didáctico integrador. Educere: Revista Venezolana de Educación, 11(39), 727-738. ISSN-e 1316-4910. (http://bit.ly/2rXJ6tO)

Arana, A. (2008). Construcción del saber institucional en torno al concepto de ambiente. Revista de Investigación, 32(63). ISSN 0798-0329 (http://bit.ly/2M54nZl).

Arana, A. (2010). Estrategias de Aprendizaje y desarrollo de conceptos. Una programación didáctica para su mediación en el aula. Revista Multidisciplinaria Dialógica, 7(1-2), 133-156. (http://bit.ly/2M97XBP).

Arana, A. (2012). Entramados... Una mirada a la complejidad. Alemania: Editorial Académica Española (http://bit.ly/36pVnpJ).

Barcedas, M. (2005). Eligiendo una estrategia de Investigación. Venezuela: Cuadernos Monográficos Candidus, (6), 34-35.

Bausela, E. (2004). La docencia a través de la Investigación-Acción. Revista Iberoamericana de Educación, 35(1), 1-9 [Revista en Línea]. (http://bit.ly/2YyLpzu).

Becerra Hernández, R., \& Moya Romero, A. (2010). Investigación-acción participativa, crítica y transformadora. Un proceso permanente de construcción. Integra Educativa, III(2). (http://bit.ly/2E56kAO)

Benbenaste, N., Luzzi, S., \& Costa, G. (2007). Vigotsky: desde el Materialismo Histórico a la Psicología. Aporte a una Teoría del sujeto del Conocimiento. [Revista en línea]. ISSN16685024 (http://bit.ly/38rCrss).

Buendía, L., Colás, P., \& Hernández, F. (2000). Métodos de Investigación en Psicopedagogía. Madrid: McGrawHill.

Colmenares, A. (2012). Investigación-acción participativa: una metodología integradora del conocimiento y la acción. Voces y Silencios: Revista Latinoamericana de Educación, 3(1), 102-115 ISSN: 2215-842114 [Revista en Línea]. (http://bit.ly/36pzNlg).

Corral, Y., Corral, I., \& Corral, A. (2016). El proceso de investigación-acción en el aula: modelo de Mckernan. Ponencia presentada en la Jornada Divulgativa de Producción Intelectual de Profesores e Investigadores de la FaCE-UC 684. Universidad de Carabobo Valencia-Venezuela. (http://bit.ly/2E2LEcu).
Hernández R., Fernández, C., \& Baptista, P. (2003). Metodología de la Investigación. México: Mc GrawHill-Interamericana.

Glaser, B., \& Strauss, A. (1967). The discovery of grounded theory. Chicago: Aldine Press.

Ley Orgánica de Educación. (2009). Gaceta oficial de la República Bolivariana de Venezuela. No 5.929 Extraordinario, de fecha 15/08/2009. (http://bit.ly/2MnoORN).

Martínez, M. (1996). Cómo hacer buen proyecto de tesis con metodología cualitativa. Revista Heterotopía. Tejiendo el pensamiento desde el otro lugar, II(2), enero-abril. Venezuela.

Martínez, M. (1999). La nueva ciencia (su desafío, lógica y método). México: Editorial Trillas.

Meira, P. (2006). Crisis ambiental y globalización: Una lectura para educadores ambientales en un mundo insostenible. (http://bit.ly/2M7spmI).

Ministerio del Poder Popular para la Educación. (2007). Currículo Nacional Bolivariano del subsistema de Educación Primaria. Caracas: Cenamec.

Moreno, C. (2012). La construcción del conocimiento: un nuevo enfoque de la educación actual. Sophia, Colección de Filosofía de la Educación, núm. 13, 2012, pp. 251-267 Universidad Politécnica Salesiana. (http://bit.ly/348FjqF).

Ochoa García, C. (2015). Consecuencias de la globalización en el manejo del medio ambiente. (http://bit.ly/2RH5rX5).

Organización de las Naciones Unidas (ONU) (2004). Resolución 59/237 de la Asamblea General de las Naciones Unidas. (http://bit.ly/36UCNWG).

Organización de las Naciones Unidas. (ONU, 2005). Documento final de la Cumbre Mundial 2005. Resultados beneficiosos en relación a la igualdad de género, resultados mixtos en relación a pobreza, paz y derechos humanos. (http://bit.ly/2S7FmAz).

Osses, S., Sánchez, I., \& Ibáñez, F. (2006). Investigación cualitativa en educación. Hacia la generación de teoría a través del proceso analítico. Estudios Pedagógicos, XXXII(1), 119-133. Universidad Austral de Chile Valdivia, Chile. (http://bit.ly/36Otsjj)

Pacheco, M. (2005). El ambiente, más allá de la Naturaleza. México: Elementos. 
Pérez, G. (1998). Investigación cualitativa. Retos e interrogantes. II Técnicas y análisis de datos. Madrid: La Muralla.

Rey Herrera, J., \& Candela, A. (2013). La construcción discursiva del conocimiento científico en el aula. Educ. Educ., 16(1), 41-65. (http://bit.ly/2PwtWnb).

United Nations Educational Scientific and Cultural Organization -UNESCO- (2012). La educación para el desarrollo sostenible en acción. (http://bit.ly/2r401uj).
United Nations Educational Scientific and Cultural Organization -UNESCO- (2015). La Educación para Todos, 2000-2015: logros y desafíos, informe de seguimiento de la EPT en el mundo.

Villegas, M. \& González, F. (2005). La construcción del conocimiento por parte de estudiantes de educación superior. Un caso de futuros docentes. Perfiles educativos, 27, 109-110, enero. México. (http://bit.ly/38qnhnn). 